\title{
Incidência e fatores de risco da retinopatia diabética em pacientes do Hospital Universitário Onofre Lopes, Natal-RN
}

\author{
Diabetic retinopathy incidence and risk factors in patients of the Onofre Lopes University \\ Hospital, Natal-RN
}

Carlos Alexandre de Amorim Garcia Alexandre Henrique Bezerra Gomes ${ }^{2}$ Israel Monte Nunes ${ }^{3}$

Tatiana Lucena de Oliveira ${ }^{3}$

Josivan Monteiro ${ }^{3}$
Trabalho realizado no Serviço de Oftalmologia do Hospital Universitário Onofre Lopes - UFRN, Natal - RN

${ }^{1}$ Coordenador da Residência Médica em Oftalmologia do Hospital Universitário Onofre Lopes (HUOL) Universidade Federal do Rio Grande do Norte (UFRN). Professor Adjunto IV da disciplina de Oftalmologia da Universidade Federal do Rio Grande do Norte - UFRN.

${ }^{2}$ Médico Residente do ${ }^{\circ}{ }^{\circ}$ ano em Oftalmologia da Universidade Federal do Rio Grande do Norte - UFRN.

${ }^{3}$ Médicos Oftalmologistas graduados pela Universidade Federal do Rio Grande do Norte - UFRN.

Endereço para correspondências: Prontoclínica de Olhos - Rua Ceará Mirim, 316, Tirol, Natal (RN)

Tel: (Fax) (84) 211-5888.

E-mail: prontoc.de.olhos@digi.com.br

Recebido para publicação em 12.07.2001

Aceito para publicação em 07.10.2002

\section{R E S U M O}

Objetivo: Estudar a incidência e fatores de risco (tempo de doença e presença de hipertensão arterial sistêmica) para retinopatia diabética em 1002 pacientes encaminhados pelo Programa de Diabetes do Hospital Universitário Onofre Lopes no período de 1992 - 1995. Métodos: Estudo retrospectivo de pacientes com diagnóstico de diabetes mellitus encaminhados ao Setor de Retina do Departamento de Oftalmologia pelo Programa de Diabetes do Hospital Universitário e submetido, sob a supervisão do autor, a exame oftalmológico, incluindo medida da acuidade visual corrigida (tabela de Snellen), biomicroscopia do segmento anterior e posterior, tonometria de aplanação e oftalmoscopia binocular indireta sob midríase (tropicamida 1\% + fenilefrina 10\%). Foi realizada análise dos prontuários referente ao tempo de doenças e diagnostico clínico de hipertensão arterial sistêmica. Resultados: Dos 1002 diabéticos examinados (em 24 deles a fundoscopia foi inviável), 978 foram separados em 4 grupos: sem retinopatia diabética (SRD), 675 casos $(69,01 \%)$; com retinopatia diabética não proliferativa(RDNP), 207 casos $(21,16 \%)$; com retinopatia diabética proliferativa (RDP), 70 casos (7,15\%); e pacientes já fotocoagulados (JFC), 26 casos $(2,65 \%)$. Do total, 291 eram do sexo masculino (29\%) e 711 do sexo feminino (71\%). Os 4 grupos foram ainda avaliados quanto ao sexo, a faixa etária, a acuidade visual, tempo de doença, presença de catarata e hipertensão arterial sistêmica e comparados entre si. Com relação ao tipo de diabetes, 95 eram do tipo $\mathrm{I}(9,4 \%), 870$ pacientes eram do tipo II $(86,8 \%)$, e em 37 casos $(3,7 \%)$ o tipo de diabetes não foi determinado. Conclusões: Comprovou-se que os pacientes com maior tempo de doença tinham maior probabilidade de desenvolver retinopatia diabética, e que a hipertensão arterial sistêmica não constituiu fator de risco em relação à diminuição da acuidade visual nos pacientes hipertensos.

Descritores:Diabetes mellitus; Retinopatia diabética/prevenção\& controle;Cuidados primários desaúde;Cegueira;Avaliaçãode programas

A retinopatia diabética (RD) é a segunda causa de cegueira irreversível, precedida apenas pela degeneração macular relacionada com a idade ${ }^{(1)}$. É a principal causa de cegueira entre 25 e 75 anos de idade ${ }^{(1-2)}$.

Duke-Elder afirma que sua evolução é previsível, porém não previnível, e relativamente intratável. Com sua evolução arrastada e progressiva, a retinopatia diabética, leva à cegueira em grande porcentagem dos casos ${ }^{(3-4)}$.

Pouco se tem estudado sobre a epidemiologia da RD no Brasil, assim 
como seus programas de prevenção da cegueira. O Programa de Diabetes do Hospital Universitário Onofre Lopes (HUOL) consiste de uma equipe multidisciplinar responsável pela orientação, acompanhamento e encaminhamento dos pacientes aos diversos profissionais da área de saúde, inclusive o oftalmologista.

O presente estudo tem o objetivo de avaliar, retrospectivamente, a incidência e os fatores de risco da retinopatia diabética (tempo de doença e presença de hipertensão arterial sistêmica) em pacientes diabéticos atendidos no ambulatório de retina do Departamento de Oftalmologia do Hospital Universitário Onofre Lopes no período de 1992 a 1995.

\section{MÉ T O D O S}

Foram examinados retrospectivamente 1002 pacientes encaminhados do Programa de Diabetes ao ambulatório de oftalmologia do HUOL no período de 1992 a 1995. Todos com diagnóstico de diabetes foram examinados no departamento de retina sob a supervisão do autor.

O exame oftalmológico constou de medida da acuidade visual corrigida (tabela de Snellen), biomicroscopia do segmento anterior e posterior, tonometria de aplanação, e fundoscopia binocular indireta sob midríase (tropicamida $1 \%+$ fenilefrina $10 \%$ ). O número de consultas variou segundo o grau da RD.

A retinopatia diabética foi classificada em $R D$ proliferativa $\mathrm{e}$ RD não proliferativa.

Foi avaliada a relação da doença com o sexo, idade, tempo estimado de diagnóstico, tipo de diabetes e retinopatia, presença clínica de hipertensão arterial sistêmica, acuidade visual e catarata.

O nível de significância escolhido foi de $5 \%$ e a análise estatística foi feita utilizando o teste do "qui-quadrado".

\section{RES U L T A D O S}

Foram examinados 2001 olhos de 1002 diabéticos (3 olhos tinham sido eviscerados). Destes, 978 tiveram pelo menos um olho examinado, e em 24 casos a fundoscopia foi inviável.

Os 978 pacientes examinados foram separados em 4 grupos: pacientes sem retinopatia diabética (SRD), correspondendo a 675 casos $(69,01 \%)$; pacientes com retinopatia diabética não proliferativa (RDNP) com $207(21,16 \%)$; pacientes com retinopatia diabética proliferativa (RDP) com 70 casos $(7,15 \%)$; e pacientes já fotocoagulados (JFC) com 26 casos $(2,65 \%)$.

Dos pacientes examinados, 291 eram do sexo masculino (29\%) e 711 do feminino (71\%) (tabela 7).

Quanto à associação entre a RD e o sexo, 187 pacientes do sexo masculino $(66,3 \%)$ eram do grupo SRD; 63 (22,3\%) tinham RDNP; 20 (7,1\%) RDP, e 12 (4,2\%) tinham fotocoagulação prévia (FP). Quanto ao sexo feminino, 488 pacientes $(70,1 \%)$ eram do grupo SRD; 144 (20,6\%) tinham RDNP; 50 (7,1\%) RDP e 14 pacientes (2\%) tinham FP (tabela 7).

Em relação à faixa etária, 147 pacientes tinham menos de 40 $\operatorname{anos}(14,7 \%), 452$ tinham entre 40 e 60 anos $(45,1 \%)$ e 345 tinham mais que 60 anos $(34,4 \%)$. Em58 casos a idade não foi catalogada $(5,8 \%)$.

Quanto à $\mathrm{RD}$ e a idade os pacientes foram divididos em 3 grupos: grupo A, formado por pacientes com idade menor ou igual a 40 anos, grupo B, formado por pacientes entre 40 e 60 anos, e grupo $\mathrm{C}$, composto por pacientes com mais de 60 anos. No grupo A, 121 pacientes $(82,8 \%)$ não apresentavam RD (grupo SRD), 17 tinham RDNP $(11,6 \%) ; 6$ RDP $(4,1 \%) ; 2$ tinham FP $(1,3 \%)$. No grupo B, 305 pacientes foram classificados como SRD (68,2\%); 103 eram portadores de RDNP (23\%); 30 pacientes tinham RDP $(6,7 \%)$; e 9 pacientes tinham FP (2\%). No grupo C, 214 pacientes foram classificados como SRD (65\%); 75 tinham RDNP (22,8\%); 27 RDP $(8,2 \%)$ e 13 pacientes tinham $\mathrm{FP}(3,9 \%)$.

De acordo com o tipo de diabetes, 95 eram do tipo I $(9,4 \%)$, 870 pacientes eram do tipo II $(86,8 \%)$, e em 37 casos $(3,7 \%)$ o tipo de diabete não foi determinado.

Dos 95 pacientes diabéticos tipo I, $62(66,7 \%)$ eram do grupo SRD; 21 (22,6\%) tinham RDNP; 9 (9,7\%) tinham RDP; 1 paciente $(1,1 \%)$ tinha fotocoagulação prévia (FP), sendo 2 não classificados. Dentre os 870 diabéticos tipo II, $593(69,8 \%)$ foram classificados como SRD; 176 (20,7\%) tinham RDNP; 57 $(6,7 \%)$ tinham RDP; e 23 pacientes $(2,7 \%)$ tinham FP, sendo 21 não classificados.

No item tempo de doença: 541 pacientes (54\%) apresentavam tempo de diabetes menor ou igual a 5 anos, $339(33,8 \%)$ tinham entre 5 e 15 anos de doença, 105 (10,5\%) tinham mais de 15 anos de doença e $17(1,7 \%)$ não tiveram o tempo de diabetes determinado. O tempo de doença variou de 1 semana a 40 anos.

Dos 53 diabéticos tipo I que apresentavam menos que 5 anos de doença, 90,6\% não tinham retinopatia diabética, enquanto $90 \%$ dos pacientes com mais de 15 anos de doença apresentavam retinopatia (Tabela 1). Quanto aos diabéticos tipo II, $81,79 \%$ dos pacientes com tempo de doença menor que 5 anos não apresentavam RD, e 61,5\% com mais de 15 anos de doença tinham retinopatia (Tabela 2 ).

A relação entre a retinopatia diabética com a hipertensão arterial sistêmica e a acuidade visual não mostrou diferenças estatisticamente significantes entre os grupos analisados (tabela 3 e 4 ).

Quanto aos pacientes que apresentavam AV menor ou igual a 20/200, $106(8,3 \%)$ não tinham RD, $44(12,2 \%)$ apresentavam RDNP, 54 (53,5\%) RDP, e 18 (43,9\%) fotocoagulação prévia. Naqueles com AV maior que 20/200, 1169 olhos $(91,7 \%)$ não

\begin{tabular}{|c|c|c|c|c|c|c|}
\hline \multirow[b]{2}{*}{ RD } & \multicolumn{2}{|c|}{$T \leq 5^{*}$} & \multicolumn{2}{|c|}{$5<T \geq 15^{*}$} & \multicolumn{2}{|c|}{$T>15^{*}$} \\
\hline & No & $\%$ & No & $\%$ & No & $\%$ \\
\hline SRD & 48 & 90,6 & 12 & 41,4 & $\mathbf{1}$ & 10 \\
\hline CRD & 5 & 9,4 & 17 & 58,6 & 9 & 90 \\
\hline TOTAL & 53 & 100 & 29 & 100 & 10 & 100 \\
\hline \multicolumn{7}{|c|}{$p>0,05(<5,>15) p<0,05(5 a 15)$} \\
\hline
\end{tabular}




\begin{tabular}{|c|c|c|c|c|c|c|}
\hline \multirow[b]{2}{*}{ RD } & \multicolumn{2}{|c|}{$\mathbf{T} \leq \mathbf{5}^{*}$} & \multicolumn{2}{|c|}{$5<T \geq 15^{*}$} & \multicolumn{2}{|c|}{$\mathrm{T}>\mathbf{1 5 *}$} \\
\hline & № & $\%$ & № & $\%$ & $\overline{\text { No }}$ & $\%$ \\
\hline SRD & 381 & 81,7 & 176 & 60,7 & 35 & 38,5 \\
\hline CRD & 85 & 18,3 & 114 & 39,3 & 56 & 61,5 \\
\hline TOTAL & 466 & 100 & 290 & 100 & 91 & 100 \\
\hline \multicolumn{7}{|c|}{$p>0,05(<5,>15) p<0,05(5 a 15)$} \\
\hline \multicolumn{7}{|c|}{$\begin{array}{l}\text { Otempo(T)foi expostoemanos; } \mathrm{RD}=\text { Retinopatia diabética; } \mathrm{SRD}=\mathrm{Sem} \\
\text { retinopatiadiabética;CRD=Comretinopatiadiabética; №-Númerodepacientes }\end{array}$} \\
\hline
\end{tabular}

\begin{tabular}{|c|c|c|c|c|}
\hline \multirow[b]{2}{*}{ RD } & \multicolumn{2}{|c|}{ ComHAS } & \multicolumn{2}{|c|}{ SemHAS } \\
\hline & No & $\%$ & No & $\%$ \\
\hline SRD & 234 & 65,5 & 441 & 71,0 \\
\hline RDNP & 80 & 22,4 & 127 & 20,5 \\
\hline RDP & 34 & 9,5 & 36 & 5,8 \\
\hline J FC & 9 & 2,5 & 17 & 2,7 \\
\hline TOTAL & 357 & 100 & 21 & 100 \\
\hline \multicolumn{5}{|l|}{$(p>0,05)$} \\
\hline
\end{tabular}

\begin{tabular}{|c|c|c|c|c|}
\hline \multirow[b]{2}{*}{ AV } & \multicolumn{2}{|c|}{ SemHAS } & \multicolumn{2}{|c|}{ ComHAS } \\
\hline & No & $\%$ & No & $\%$ \\
\hline$A V \leq 20 / 200$ & 78 & 24,1 & 47 & 20,5 \\
\hline$A V>20 / 200$ & 246 & 75,9 & 183 & 79,5 \\
\hline TOTAL & 324 & 100 & $\mathbf{2 3 0}$ & 100 \\
\hline \multicolumn{5}{|c|}{ Emnúmerodeolhos/p>0,05 } \\
\hline \multicolumn{5}{|c|}{ №=Númerodepacientes } \\
\hline
\end{tabular}

\begin{tabular}{|c|c|c|c|c|}
\hline \multirow[b]{2}{*}{ RD } & \multicolumn{2}{|c|}{$A V \leq 20 / 200$} & \multicolumn{2}{|c|}{$A V>20 / 200$} \\
\hline & № & $\%$ & No & $\%$ \\
\hline SRD & 106 & 8,3 & 1169 & 91,7 \\
\hline RDNP & 44 & 12,2 & 316 & 87,8 \\
\hline RDP & 54 & 53,5 & 47 & 46,5 \\
\hline J FC & 18 & 43,9 & $\mathbf{2 3}$ & 56,1 \\
\hline \multicolumn{5}{|l|}{$(p>0,05)$} \\
\hline \multicolumn{5}{|c|}{$\begin{array}{l}\text { RD=Retinopatiadiabética;SRD=SemRD;RDNP=RDnãoproliferativa; } \\
\text { RDP=RDproliferativa;J FC=J áfotocoagulado; Nodepacientes }\end{array}$} \\
\hline
\end{tabular}

apresentavam RD, $316(87,8 \%)$ tinham RDNP, 47 (46,5\%) RDP, e $23(56,1 \%)$ fotocoagulação prévia (Tabela 5).

A presença de catarata foi observada em $49,7 \%$ dos pacientes sem RD, em 55,5\% dos casos com RDNP, em 65,7\% dos pacientes com RDP; $65,2 \%$ dos pacientes já fotocoagulados apresentavam catarata (Tabela 6).

A fotocoagulação a laser foi realizada em 146 pacientes

\begin{tabular}{|c|c|c|c|c|}
\hline \multirow[b]{2}{*}{ RD } & \multicolumn{2}{|c|}{ Comcatarata } & \multicolumn{2}{|c|}{ Semcatarata } \\
\hline & № & $\%$ & No & $\%$ \\
\hline SRD & 336 & 49,7 & 339 & 50,3 \\
\hline RDNP & 115 & 55,5 & $\mathbf{9}$ & 44,5 \\
\hline RDP & 46 & 65,7 & 24 & 34,3 \\
\hline J FC & 18 & 69,2 & 8 & 30,8 \\
\hline \multicolumn{5}{|c|}{ Olhos $(p>0,05)$} \\
\hline \multicolumn{5}{|c|}{$\begin{array}{l}\text { RD=Retinopatia diabética;SRD=Sem RD;RDNP=RDnãoproliferativa; } \\
\text { RDP =RDproliferativa;J FC =J áfotocoagulado; No=Númerodepacientes }\end{array}$} \\
\hline
\end{tabular}

(14,9\%), e em $832(85,1 \%)$ esta não foi indicada. Quanto ao tipo de RD, em 70 pacientes $(33,8 \%)$ com RDNP o laser foi realizado, e em 137 (66,2\%) não houve indicação. Todos os pacientes portadores de RDP foram fotocoagulados com laser de argônio.

D I S CUS S ÃO

O diabetes afeta aproximadamente nove milhões de brasileiros e lesa vários órgãos, incluindo os rins, os nervos periféricos e, particularmente, os olhos ${ }^{(2)}$.

O maior número de pacientes femininos neste estudo $(71 \%)$, não corresponde à incidência de diabetes neste grupo segundo trabalhos publicados, os quais revelam uma proporção de 3:4 respectivamente para o sexo masculino e feminino ${ }^{(3)}$ (tabela 7).

No grupo de pacientes com idade abaixo de 40 anos houve uma menor incidência de retinopatia diabética, e não houve diferença estatisticamente significativa quanto à retinopatia nos grupos dos pacientes com idade entre 40 e 60 anos e idade maior que 60 anos, o que confirma os resultados publicados em outros trabalhos ${ }^{(6)}$.

Ficou evidenciado nas tabelas 1 e 2 , que os pacientes com maior tempo de diabetes tinham mais probabilidade de desenvolver a RD, principalmente os diabéticos tipo I, onde $90 \%$ dos pacientes com mais de 15 anos de doença apresentavam retinopatia diabética, fato concordante com a literatura que refere estar a incidência da $\mathrm{RD}$ intimamente associada à duração da doença ${ }^{(7-8)}$. Em relação ao tipo de diabetes, não houve diferença significativa quanto às alterações encontradas.

Neste estudo, a HAS não pareceu constituir fator agravante para a retinopatia diabética (Tabela 3), indo de encontro com alguns trabalhos publicados ${ }^{(8-9)}$. A hipertensão arterial é considerada um fator de risco importante para a retinopatia diabética, principalmente em pacientes com nefropatia ${ }^{(2,8)}$.

Em relação à $\mathrm{AV}$, observou-se que a presença de HAS na RD não afeta a AV (Tabela 4), e que esta se encontrava bastante afetada pela presença de RD, RDNP, RDP e FP.

Foi demonstrado que quanto mais severa o tipo de retinopatia, maior a incidência de catarata (Tabela 6), com conseqüente piora da acuidade visual.

O maior número de pacientes $(69 \%)$ sem retinopatia diabética deste trabalho, ao contrário de outros estudos ${ }^{(1,8-9)}$ em que 


\begin{tabular}{|ccccc|}
\hline \multicolumn{4}{|c|}{ Tabela 7. Relaçãoentre númerode pacientes porsexo, retinopatia diabéticaefotocuagulaçãoanterior } \\
Sexo & Sem RD & RDNP & RDP & Fotocoagulaçãoanterior \\
291 homens (29\%) & $187(66,3 \%)$ & $63(22,3 \%)$ & $\mathbf{2 0}(7,1 \%)$ & $12(4,2 \%)$ \\
711 mulheres (71\%) & $488(70,1 \%)$ & $144(20,6 \%)$ & $50(7,1 \%)$ & $14(2,0 \%)$ \\
RD=Retinopatiadiabética;RDNP=RDnãoproliferativa;RDP=RDproliferativa & & \\
\hline
\end{tabular}

predomina a presença de retinopatia, deve-se ao trabalho de esclarecimento e assistência médica, desenvolvido há vários anos pelo Programa de Diabetes, juntamente com o Serviço de Oftalmologia do HUOL.

\section{ONCLUS ̃̃O}

Comprovou-se que os pacientes com maior tempo de doença tinham maior probabilidade de desenvolver retinopatia diabética e que aqueles pacientes com hipertensão arterial sistêmica não constituiu fator de risco para diminuição da acuidade visual.

Os Programas de Diabetes nos hospitais públicos e privados são pontos essenciais na prevenção da cegueira ${ }^{(2-3,5)}$, onde os pacientes são acompanhados por uma equipe multidisciplinar, visando o diagnóstico precoce da doença; no nosso caso, isto contribuiu para diminuição da incidência de retinopatia diabética pelo diagnóstico precoce, e assim diminuindo o número de deficientes visuais devido ao diabetes.

\section{A B S T R A C T}

Purpose: To study the incidence and risk factors (length of illness and arterial hypertension) for diabetic retinopathy in 1002 patients who were submitted to the diabetes program at the Onofre Lopes University Hospital from 1992 to 1995. Methods: Retrospective study of patients diagnosed with diabetes mellitus referred by the university's diabetes Program to the Retina Department and were submitted to an ophthalmological examination, under the author's supervision, including: measurement of visual acuity, anterior and posterior biomicroscopy, Goldman applanation tonometry, and indirect ophthalmoscopy (tropicamide 1\% + phenylephrine 10\%) and analysis of the patients' records regarding length of disease and clinical arterial hypertension diagnosis. Results: Of 1002 diabetic patients (in 24 fundoscopy was impossible to perform)
978 were divided into 4 groups: without diabetic retinopathy, 675 cases $(69,01 \%)$; with non proliferative diabetic retinopathy, 207 cases $(21,16 \%)$; with proliferative diabetic retinopathy, 70 cases $(7,15 \%)$; and photocoagulated patients, 26 cases $(2,65 \%)$. Of the total, 291 were males $(29 \%)$ and 711 females $(71 \%)$. These 4 groups were analyzed regarding gender, age, visual acuity, duration of the disease, presence of cataract and systemic hypertension.Concerning the type of diabetes, 95 were type I $(9,4 \%), 870$ were type II $(86,8 \%)$, and in 37 cases $(3,7 \%)$ the type of diabetes was not determined. Conclusion: It was shown that the longer patients had the disease, more likely they were to develop diabetic retinopathy and that hypertension systemic did not constitute a risk factor for the decrease of visual acuity in the hypertensive patients.

Keywords: Diabetes mellitus; D iabetic retinopathy/prevention \& control; Primary health care; Blindness; Program evaluation

\section{REFERENCIAS}

1. Vilela MP, Saadi AK, Pletsch L, Giacomet A. Inquérito entre pacientes e médicos sobre as estratégias aplicadas na prevenção e tratamento da retinopatia diabética. Arq Bras Oftalmol 1997;60:152-5.

2. Nehemy MB. Retinopatia diabética. Arq Bras Oftalmol 1998;61:366-70.

3. Duke-Elder S. Diabetic Retinopathy. In: Duke-Elder S. System of Ophthalmology. London: Henry Kimpton; 1967. p.410-48.

4. Hirata CA, Fang T, Casella AMB, Elieser M, Abujamra S. Prevalência de retinopatia em uma população de diabéticos. Arq Bras Oftalmol 1986;49:31-3.

5. Barros OM. Analyse de la frequence de retinopathie diabétique quant au sexe,âge et temps de durée de la maladie: étude de 563 cas. In: XXII.Concilium Ophtalmologicum. Paris: Masson; 1974. p.530-8.

6. Caird FI.. Epidemiology of diabetic retinopathy. In: Lynn JR, Snyder WB, Vaiser A., editors. Diabetic retinopathy. New York: Grune e Stratton; 1974.

7. Ulson ACB, Abujamra S. Análise de 400 pacientes diabéticos portadores de retinopatia. Arq Bras Oftalmol 1986;49:9-12.

8. Steck AD, Esteves RF, Gonçalves JCM. Prevalência de retinopatia diabética em uma população de diabéticos do HC de Franco da Rocha-SP. Arq Bras Oftalmol 1993;56:125-8.

9. Casella AMB, Bonomo PP, Farah ME. Prevalência da retinopatia diabética em pacientes diabéticos, atendidos pelo Sistema Único de Saúde(SUS), de Londrina-Paraná. Arq Bras Oftalmol 1994;57:5-9.

Nos artigos enviados para publicação, o nome dos autores e suas afiliações devem estar completos. Isso facilitará a indexação e os links com as bases de dados e o CV Lates. 